\title{
PARTITIONING PAIRS OF COUNTABLE SETS
}

\author{
STEVO TODORCEVIC
}

(Communicated by Andreas R. Blass)

\begin{abstract}
We make the translations of our partitions from [3] in the context of all countable subsets of a fixed uncountable set. A different translation was obtained recently by Velleman [4].
\end{abstract}

The purpose of this paper is to define a two-cardinal version of one of our partitions from [3].

Theorem. For every uncountable set $A$ there is a $c:\left[[A]^{\aleph_{0}}\right]^{2} \rightarrow A$ such that, for every cofinal $U \subseteq[A]^{\aleph_{0}}$ and $\alpha$ in $A$, there exist $x \subset y$ in $U$ such that $c(x, y)=\alpha$.

The proof will use straightforward generalization of one of the partitions from [3]. We shall assume that $A$ is equal to some initial ordinal $\theta$, and we shall fix an

$$
r:[\theta]^{\aleph_{0}} \rightarrow\{0,1\}^{\omega}
$$

such that $r_{x} \neq r_{y}$ for $x \subset y$. [Identifying $\omega_{1}$ with a subset of $\{0,1\}^{\omega}$, let $r_{x}$ (including finite $x$ ) be the standard code of $\left(\operatorname{tp} x, q_{x}\right)$, where $q_{x}$ is defined recursively on $\sup x$ as follows assuming that, for each ordinal $\alpha$ of cofinality $\omega$, we have a fixed increasing sequence $\left\{\alpha_{i}\right\}$ converging to $\alpha$ : If $x$ has a maximal element $\xi$ set $q_{x}(0)=1$ and $q_{x}(i+1)=r_{y}(i)$, where $y=x \cap \xi$. If $\alpha=\sup x$ is a limit ordinal, let $q_{x}(0)=0$ and $q_{x}\left(2^{i}(2 j+1)\right)=r_{x_{i}}(j)$, where $x_{i}=x \cap \alpha_{i}$.] Moreover, we shall fix a one-to-one $e_{x}: x \rightarrow \omega$ for each $x$ in $[\theta]^{\aleph_{0}}$. For an integer $n$ and $x$ in $[\theta]^{\aleph_{0}}$, we set

$$
x(n)=\left\{\xi \in x: e_{x}(\xi) \leq n\right\} .
$$

For $x \subset y$ in $[\theta]^{\aleph_{0}}$, let

$$
\Delta(x, y)=\Delta\left(r_{x}, r_{y}\right) \text {, }
$$

i.e., the minimal place where the reals $r_{x}$ and $r_{y}$ disagree. Finally, for $x \subset y$ in $[\theta]^{\kappa_{0}}$ and an ordinal $\lambda \leq \theta$, we set

$$
c_{\lambda}(x, y)=\min (y(\Delta(x, y)) \backslash \sup (x \cap \lambda)),
$$

Received by the editors October 10, 1989 and, in revised form, February 9, 1990. 1980 Mathematics Subject Classification (1985 Revision). Primary 03E05, 04A20.

Research at MSRI supported in part by NSF Grant DMS-8505550. 
i.e., $c_{\lambda}(x, y)$ is the minimal ordinal of $y(\Delta(x, y))$ which is bigger than or equal to the supremum of $x \cap \lambda$; if this set is empty, set $c_{\lambda}(x, y)=0$.

To state the basic property of our partitions $c_{\lambda}$, let $\lim (\omega)$ denote the class of all ordinals of cofinality $\omega$.

Lemma. Suppose $\kappa \neq \lambda$ are regular uncountable cardinals $\leq \theta$ and that $S$ and $T$ are stationary subsets of $\lim (\omega) \cap \kappa$ and $\lim (\omega) \cap \lambda$, respectively. Then, for every cofinal $U \subset[\theta]^{\aleph_{0}}$, there exist $x \subset y$ in $U$ such that $c_{\kappa}(x, y) \in S$ and $c_{\lambda}(x, y) \in T$.

Proof. Choose a countable elementary submodel $M$ of $H_{\left(2^{\theta}\right)^{+}}$containing all relevant objects such that $\gamma=\sup (M \cap \kappa)$ is in $S$ and $\delta=\sup (M \cap \lambda)$ is in $T$. [If $\kappa<\lambda$, first pick submodel $N$ such that $N \cap \lambda$ is in $T$, then pick a countable submodel $\bar{M}$ containing $N$ as an element such that $\sup (\bar{M} \cap \kappa)$ is in $S$ and set $M=\bar{M} \cap N$.] Choose a $y$ in $U$ containing $(M \cap \theta) \cup\{\gamma, \delta\}$ and an integer $n$ such that both $\gamma$ and $\delta$ are in $y(n)$. For $s$ in $\{0,1\}^{<\omega}$, let $U_{s}$ be the set of all $z$ in $U$ such that $r_{z}$ extends $s$. Let $\Sigma$ be the set of all $s$ in $\{0,1\}^{<\omega}$ for which $U_{s}$ is cofinal. Note that, by elementarity of $M$, all restrictions of $r_{y}$ are in $\Sigma$. Since $r_{y}$ is not in $M$ there must be an $s$ in $\Sigma$ which splits from $r_{y}$ at some place $m \geq n$. Pick $\alpha$ in $M \cap \kappa$ above every element of $y(m) \cap \gamma$, and also pick $\beta$ in $M \cap \lambda$ above every element of $y(m) \cap \delta$. Since $U_{s}$ is cofinal and since it is an element of $M$, we can find an $x$ in $U_{s} \cap M$ containing $\alpha$ and $\beta$. Then $\Delta(x, y)=m$, and $\gamma$ and $\delta$ are minimal ordinals of $y(m)$ above $\sup (x \cap \kappa)$ and $\sup (x \cap \lambda)$, respectively. This finishes the proof.

Now the theorem follows easily: If $\theta$ is a regular cardinal we let $c$ be the composition of $c_{\theta}$ with a splitting of $\lim (\omega) \cap \theta$ into $\theta$ disjoint stationary sets. If $\theta$ is singular, let $\kappa$ be the maximum of $\left\{\omega_{1}, \operatorname{cf} \theta\right\}$ and let $S_{\xi}, \xi<\operatorname{cf} \theta$, be a partition of $\lim (\omega) \cap \kappa$ into disjoint stationary sets. Let $\lambda_{\xi}, \xi<\operatorname{cf} \theta$, be an increasing sequence of regular cardinals converging to $\theta$ with $\lambda_{0}>\kappa$. For each $\xi$ fix a partition $T_{\xi}^{\eta}, \eta<\lambda_{\xi}$, of $\lim (\omega) \cap \lambda_{\xi}$ into disjoint stationary sets. Finally, for $x \subset y$ in $[\theta]^{\aleph_{0}}$, we let $c(x, y)$ be equal to $\langle\xi, \eta\rangle$ if $c_{\kappa}(x, y) \in S_{\xi}$ and $c_{\lambda_{\xi}}(x, y) \in T_{\xi}^{\eta}$. By the lemma, every $\langle\xi, \eta\rangle$ is realized in every cofinal $U \subseteq[\theta]^{\aleph_{0}}$.

By looking at some other partitions of [3] it is natural to ask whether we can define $c(x, y)$ to be an element of $[\theta]^{\aleph_{0}}$ rather than an ordinal from $\theta$ with the hope that the set of values of $c$ on the square of every cofinal $U \subseteq[\theta]^{\aleph_{0}}$ contains a closed and unbounded set. Unfortunately, such a hope cannot be realized since there might be an unbounded subset of $[\theta]^{\aleph_{0}}$ of smaller size than any closed and unbounded set in $[\theta]^{\aleph_{0}}$. In principle, it is still possible to have a stationary set $S \subset[\theta]^{\kappa_{0}}$ such that every cofinal $U \subset[\theta]^{\kappa_{0}}$ realizes every color from a closed and unbounded set restricted to $S$. This is the approach taken by Velleman [4]. Unfortunately, [4] assumes a too strong fact about 
$[\theta]^{\aleph_{0}}$, the existence of a stationary set of size $\theta$, which rules out many $\theta$ 's, in particular, all $\theta$ 's of cofinality $\omega$. A more relaxed approach goes as follows. Let stationary cofinality of $[A]^{\aleph_{0}}$ be the minimal cardinality of a stationary subset of $[A]^{\aleph_{0}}$. (We do not know whether the stationary cofinality of $[A]^{\aleph_{0}}$ is, in fact, equal to the cofinality of $[A]^{\aleph_{0}}$.) A Kurepa family in $[A]^{\aleph_{0}}$ is a family $K$ of countable subsets of $A$ such that the union of every uncountable subfamily of $K$ is uncountable. Let $K$ be a Kurepa family in $[A]^{\aleph_{0}}$, and let $A^{*}$ be the set of all finite sequences of elements of $A$. For each $k$ in $K$ fix an onto map $f_{k}: \omega \rightarrow k$, and let $k^{*}$ be the set of all proper initial subsequences of $f_{k}$. Then $K^{*}=\left\{k^{*}: k \in K\right\}$ is a Kurepa family on $A^{*}$ in the usual sense [2], i.e., for every $a$ in $\left[A^{*}\right]^{\aleph_{0}}$, there exist only countably many sets of the form $k^{*} \cap a$ for $k^{*}$ in $K^{*}$.

Theorem. Assume $S$ is stationary in $[A]^{\aleph_{0}}$ and that there is a Kurepa family in $[A]^{\aleph_{0}}$ of size $S$. Then there is a $d:\left[[A]^{\aleph_{0}}\right]^{2} \rightarrow[A]^{\aleph_{0}}$ such that, for every cofinal $U \subset[A]^{\aleph_{0}}$, there is a closed and unbounded set of elements of $S$ of the form $d(x, y)$ for some $x \subset y$ in $U$.

Fix one-to-one $i: S \rightarrow[A]^{\aleph_{0}}$ such that $i^{\prime \prime} S$ is a Kurepa family in $[A]^{\aleph_{0}}$. For each $y$ in $[A]^{\aleph_{0}}$, we let

$$
S_{y}=\{x \in S: x \cup i(x) \subseteq y\}
$$

and fix $e_{y}: S_{y} \rightarrow \omega$ one-to-one. For an integer $n$ and $y$ in $[A]^{\aleph_{0}}$, set

$$
y(n)=\left\{x \in S_{y}: e_{y}(x) \leq n\right\} .
$$

Finally, for $x \subset y, d(x, y)$ is the $\subseteq$-minimal element of $y(\Delta(x, y))$ containing $x$, if such an element exists; otherwise $d(x, y)=y$. It is now quite easy to check that $d$ works.

Since the family of singletons of elements of $A$ is clearly a Kurepa family in $[A]^{\aleph_{0}}$, the hypothesis of the second theorem is satisfied when the stationary cofinality of $[A]^{\aleph_{0}}$ is equal to the size of $A$. It is well known that, besides the infinite products of infinite sets and their finite successors, this is true for $A$ of size $<\aleph_{\omega}$ (see [1]), or if the size of $A$ has uncountable cofinality provided $0 \#$ does not exist. Thus, left open is the case $A=\theta$ and $\operatorname{cf} \theta=\omega$. In [2, $\S 8]$ (see also [3, §1]), we have produced a Kurepa family in $[\theta]^{\aleph_{0}}$ of size $\theta^{+}$ assuming $\square_{\theta}$. Thus, if, moreover, the stationary cofinality of $[\theta]^{\aleph_{0}}$ is equal to $\theta^{+}$, the hypothesis of the second theorem is satisfied. All this shows that, for example, the constructible universe is the natural model which satisfies this hypothesis for every set $A$. Note that some such hypothesis is needed here since, for example, the Chang's Conjecture for $\left(\aleph_{\omega+1}, \aleph_{\omega}\right)$ easily implies that every subset of $\left[\aleph_{\omega}\right]^{\aleph_{0}}$ of size $>\aleph_{\omega}$ contains an uncountable subset with countable union.

In the case $A=\theta$ and $\operatorname{cf} \theta=\omega$, the number of colors given by the partition $d$ is larger than $\theta$. This follows from the fact that if $K$ is a Kurepa family in 
$[A]^{\aleph_{0}}$, then every stationary set $S$ in $[A]^{\aleph_{0}}$ splits into $K$ disjoint stationary sets. To see this, for every $x$ in $[A]^{\aleph_{0}}$, fix one-to-one

$$
f_{x}:\{k \in K: k \subseteq x\} \rightarrow \omega,
$$

and, for $n$ in $\omega$ and $k$ in $K$, define

$$
U_{k}^{n}=\left\{x \in[A]^{\aleph_{0}}: k \subseteq x \text { and } f_{x}(k)=n\right\} .
$$

Then for a fixed $k, U_{k}^{n}$ cover the supersets of $k$ while, for a fixed $n, U_{k}^{n}$ are disjoint. Thus we have an analogue of the Ulam matrix and we can proceed as in the classical case. (If the size of $K$ has countable cofinality, we first split $S$ into countably many disjoint stationary sets $S_{i}$ and then split each $S_{i}$ into a sufficient number of disjoint stationary sets.)

Note that if $K$ is a Kurepa family in $[A]^{\aleph_{0}}$ containing all singletons, then

$$
F(x)=\{k \in K: k \subseteq x\}
$$

is an isomorphical embedding of $[A]^{\aleph_{0}}$ into a cofinal subset of $[K]^{\aleph_{0}}$. Conversely, if $[\theta]^{\aleph_{0}}$ and $[\lambda]^{\aleph_{0}}$ (where $\theta \leq \lambda$ ) are cofinally similar (see [1]), then there is a Kurepa family in $[\theta]^{\aleph_{0}}$ of size $\lambda$. To see this pick a Tukey function $H:[\lambda]^{\aleph_{0}} \rightarrow[\theta]^{\aleph_{0}}$, i.e., a function mapping unbounded sets into unbounded sets. Then $\{H(\{\xi\}): \xi<\lambda\}$ is a Kurepa family in $[\theta]^{\aleph_{0}}$ of size $\lambda$.

The basic result of this paper is true when $[A]^{\kappa_{0}}$ is replaced by $[A]^{\kappa}$ for every regular $\kappa$. This is proved using an analogue of the partition $a$ of $[3, \S 5]$ : Given $x \subset y$ in $[A]^{\kappa}$ with $\sup (x)$ in $y$, one first walks inside $y$ (along a fixed $c$-sequence on $\kappa^{+}$and modulo the isomorphism of $y$ and its order type) from $\sup (y)$ to $\sup (x)$ and then picks the first place where the corresponding closed and unbounded sets have made a non-trivial oscillation provided the maximum of their intersection is in $x$.

\section{REFERENCES}

1. J. R. Isbell, The category of cofinal types II, Trans. Amer. Math. Soc. 116 (1965), 394-416.

2. S. Todorcevic, Trees and linearly ordered sets, Handbook of Set-Theoretic Topology (K. Kunen and J. Vaughan, eds.), North-Holland, 1984.

3. __ Partitioning pairs of countable ordinals, Acta Math. 159 (1987), 261-294.

4. D. Velleman, Partitioning pairs of countable sets of ordinals, preprint.

Mathematical Institute, Belgrade, Yugoslavia

Current address: Mathematical Sciences Research Institute, 1000 Centennial Drive, Berkeley, California 94720 